\title{
Gua Lou Gui Zhi decoction suppresses LPS-induced activation of the TLR4/NF-кB pathway in BV-2 murine microglial cells
}

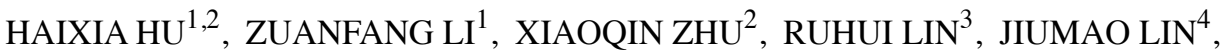 \\ JUN PENG ${ }^{3}$, JING TAO ${ }^{1,2}$ and LIDIAN CHEN ${ }^{1-3}$
}

\begin{abstract}
${ }^{1}$ Key Laboratory of TCM Rehabilitation of the State Administration of Traditional Chinese Medicine; ${ }^{2}$ Fujian Rehabilitation Technology Engineering Center; ${ }^{3}$ MOE Key Laboratory of Traditional Chinese Medicine on Osteology and Traumatology and Exercise Rehabilitation; ${ }^{4}$ Fujian Key Laboratory of Exercise Rehabilitation, Fujian University

of Traditional Chinese Medicine, Minhou Shangjie, Fuzhou, Fujian 350122, P.R. China
\end{abstract}

Received January 18, 2013; Accepted March 19, 2013

DOI: $10.3892 /$ ijmm.2013.1331

\begin{abstract}
Toll-like receptor 4 (TLR4)/nuclear factor- $\kappa \mathrm{B}$ $(\mathrm{NF}-\kappa \mathrm{B})$ signaling-mediated neuroinflammation contributes to secondary brain damage in ischemic stroke; therefore, antiinflammatory therapy via suppression of the TLR4/NF- $\kappa B$ pathway could be a promising strategy for the treatment of stroke and post-stroke disabilities. Gua Lou Gui Zhi decoction (GLGZD) has long been used in China to clinically treat dysfunction after stroke such as muscular spasticity, but the precise mechanisms are largely unknown. In the present study, we evaluated the anti-inflammatory effect of GLGZD and investigated the underlying molecular mechanisms using lipopolysaccharide (LPS)-stimulated BV-2 microglial cells as an in vitro inflammatory model of neural cells. We found that GLGZD inhibited the inflammatory response in microglial cells as it significantly reduced LPS-induced expression of pro-inflammatory nitric oxide, tumour necrosis factor- $\alpha$, interleukin (IL)- 6 and IL-1 $\beta$ in BV-2 cells, in a dose-dependent manner. In addition, GLGZD treatment significantly decreased the protein expression of TLR4 and myeloid differentiation factor 88 , inhibited the phosphorylation of $\mathrm{I} \kappa \mathrm{B}$ and blocked the nuclear translocation of NF- $\kappa \mathrm{B}$ in BV-2 cells, demonstrating its inhibitory effect on the activation of TLR4/NF- $\mathrm{B}$
\end{abstract}

Correspondence to: Dr Jing Tao or Professor Lidian Chen, College of Rehabilitation Medicine, Fujian University of Traditional Chinese Medicine, 1 Huatuo Road, Minhou Shangjie, Fuzhou, Fujian 350108, P.R. China

E-mail: taojing01@yahoo.com.cn

E-mail: cld@fjtcm.edu.cn

Abbreviations: GLGZD, Gua Lou Gui Zhi decoction; CNS, central nervous system; LPS, lipopolysaccharide; NO, nitric oxide; iNOS, inducible NO synthase; IL-1 $\beta$, interleukin-1 $\beta$; TLR4, Toll-like receptor 4; TNF- $\alpha$, tumour necrosis factor- $\alpha$; NF- $\kappa \mathrm{B}$, nuclear factor- $\kappa \mathrm{B}$; MyD88, myeloid differentiation factor 88 ; I $\kappa \mathrm{B} \alpha$, inhibitor of $\kappa \mathrm{B} \alpha$

Key words: Traditional Chinese Medicine, Gua Lou Gui Zhi decoction, $\mathrm{NF}-\kappa \mathrm{B}$, inflammation, stroke signaling. Collectively, our findings suggest that inhibition of the inflammatory response via suppression of the TLR4/ $\mathrm{NF}-\kappa \mathrm{B}$ pathway may be one of the mechanisms through which GLGZD ameliorates the damage in ischemic cerebral tissues.

\section{Introduction}

Stroke is the second most common cause of death and the major cause of disability worldwide (1). Inflammatory reaction has been shown to play an important role in secondary brain damage after stroke, which is believed to be the consequence of microglial activation (2,3). Microglial cells are generally considered to be the resident immunocompetent cells of the central nervous system (CNS), and over-activation of microglia can induce significant and highly detrimental neurotoxic effects through excessive production of a large array of cytotoxic factors such as superoxide, nitric oxide (NO), tumour necrosis factor- $\alpha$ (TNF- $\alpha$ ), interleukin (IL)- $1 \beta$ and IL-6 (4). In addition, previous studies have demonstrated that a decrease in the secretion of pro-inflammatory mediators in microglia attenuates the severity of neuro-degenerative diseases including ischemic stroke $(5,6)$. LPS, an inducer of inflammation, has been frequently employed to stimulate microglia to construct a useful model for the study of mechanisms that underlie neuronal injury (7). Toll-like receptor 4 (TLR4) is well known as the unique receptor for lipopolysaccharide (LPS), which transduces immune-related signals to the nucleus via activating transcription factors such as nuclear factor- $\kappa \mathrm{B}(\mathrm{NF}-\kappa \mathrm{B})$. In unstimulated cells, $\mathrm{NF}-\kappa \mathrm{B}$ is sequestered in the cytosol via interaction with inhibitory $\mathrm{I} \kappa \mathrm{B}$ proteins. However, when cells receive pathological stimuli, I $\mathrm{B}$ protein is phosphorylated resulting in its ubiquitination and degradation, which in turn releases sequestered $N F-\kappa B$, leading to its translocation to the nucleus where it positively regulates the expression of various pro-inflammatory mediators (8-10). Therefore, anti-inflammatory treatment may reduce ischemic brain injury and enhance stroke recovery.

Gua Lou Gui Zhi decoction (GLGZD) is a classical traditional Chinese formula that was first prescribed in Eastern Han Dynasty, around 210 AD, which consists of a combination of six herbs, including Trichosanthis Radix, Ramulus Cinnamomi, 
Paeonia lactiflora, Glycyrrhiza, Zingiber officinale Roscoe and Fructus Jujubae. GLGZD has long been used in China to clinically treat post-stroke disabilities such as muscular spasticity (11-13). However, the mode of its action remains largely unclear. Using LPS-stimulated microglial BV-2 cells as an in vitro inflammatory model of neural cells, in the present study we evaluated the anti-inflammatory effect of GLGZD and investigated the underlying molecular mechanisms.

\section{Materials and methods}

Materials and reagents. LPS (from Escherichia coli 055:B5) and 3-[4,5-dimethyl-2-thiazolyl]-2,5-diphenyltetrazolium bromide (MTT) were obtained from Sigma-Aldrich (St. Louis, MO, USA). Fetal bovine serum (FBS), Dulbecco's modified Eagle's medium (DMEM), penicillin, streptomycin, 0.05\% (w/v) trypsin/EDTA, and phosphate-buffered saline (PBS) were obtained from Gibco-BRL (Gaithersburg, MD, USA). Cytokine (IL-6, TNF- $\alpha$ and IL-1 $\beta$ ) ELISA kits were purchased from R\&D Systems (Minneapolis, MN, USA). Antibodies for western blot analysis included: i) TLR4 antibody (rodentspecific) rabbit polyclonal antibody; ii) MyD88 (D80F5) rabbit $\mathrm{mAb}$; iii) inhibitor of $\kappa \mathrm{B} \alpha(\mathrm{I} \kappa \mathrm{B} \alpha)(\mathrm{L} 35 \mathrm{~A} 5)$ mouse $\mathrm{mAb}$ (aminoterminal antigen); iv) phospho-IкB $\alpha$ (Ser32/36) (5A5) mouse $\mathrm{mAb}$; v) anti- $\beta$-actin (8H10D10) mouse $\mathrm{mAb}$; vi) horseradish peroxidase (HRP)-conjugated secondary antibodies (all from Cell Signaling Technology, Beverly, MA, USA). The antibody for immunofluorescence was NF-kB p65 (F-6) (Santa Cruz Biotechnology, Inc., Santa Cruz, CA, USA).

Preparation of water extract from GLGZD. Medicinal plants were supplied by Guo Yi Tang Chinese Herbal Medicine Store (Fujian, China) for the preparation of the GLGZD water extract. The preparation was a mixture of six crude plant extract ingredients soaked in (5-fold of the plant) double distilled water (DDW) for $30 \mathrm{~min}$. The mixture was heated to $100^{\circ} \mathrm{C}$ for $1 \mathrm{~h}$, and the decoction was filtrated. The filtrates obtained from two cycles of the procedures were mixed, then filtered and concentrated using a rotary evaporator (Model RE-2000; Yarong Biochemistry Instrument Co., Shanghai, China). The extract of GLGZD was obtained by a spraying desiccation method using a spray dryer (Model B-290; Büchi Labortechnik AG, Flawil, Switzerland). The stock and working concentrations of GLGZD were prepared by dissolving the extract in culture media to a concentration of $50 \mathrm{mg} / \mathrm{ml}$ and $50,100,200 \mu \mathrm{g} / \mathrm{ml}$.

Cell culture and treatment. A murine BV-2 microglial cell line [purchased from the American Type Culture Collection, Manassas, VA, USA] was maintained in DMEM supplemented with FBS (10\%), $100 \mathrm{U} / \mathrm{ml}$ penicillin and $100 \mu \mathrm{g} / \mathrm{ml}$ streptomycin. Cells were incubated in culture medium at $37^{\circ} \mathrm{C}$ in a $95 \%$ atmospheric air and $5 \% \mathrm{CO}_{2}$ humidified atmosphere. Briefly, the numbers of viable cells were evaluated by counting trypan blue-excluding cells that were then plated at a density of $1 \times 10^{5}$ cells/well in 96-well trays, $2 \times 10^{5}$ cells/well in 24 -well trays, or plated at a density of $5 \times 10^{5}$ cells/well in 6 -well trays (for remaining experiments), incubated at $37^{\circ} \mathrm{C}$ for $24 \mathrm{~h}$, and given a fresh change of medium. Cells were then incubated with or without LPS $(1 \mu \mathrm{g} / \mathrm{ml})$ in the presence of various concentrations of GLGZD $(50,100,200$ or $500 \mu \mathrm{g} / \mathrm{ml})$ at $37^{\circ} \mathrm{C}$ for an additional $24 \mathrm{~h}$, and in all experiments, cells were serum starved for $4 \mathrm{~h}$ prior to the treatment. The cultured cells were treated with LPS with or without GLGZD for $24 \mathrm{~h}$ (for ELISA), or for $12 \mathrm{~h}$ (for RT-PCR), or for $1 \mathrm{~h}$ for NF- $\mathrm{kB}$ translocation activity and western blotting for relative protein expression.

Cytotoxity assay. Microglial BV-2 cells were grown in 96-well plates and then incubated with or without LPS $(1 \mu \mathrm{g} / \mathrm{ml})$ in the presence of GLGZD at various concentrations for $24 \mathrm{~h}$. MTT assay was used to measure the viability of the cells. MTT is a pale yellow substrate that produces a dark blue formazan product when incubated with living cells; an MTT ring is cleaved in active mitochondria, which occurs only in living cells. After the supernatants were removed for nitrite determination, cells were incubated at $37^{\circ} \mathrm{C}$ with MTT $(0.05 \mathrm{mg} / \mathrm{ml})$ for $4 \mathrm{~h}$. The resulting color was assayed at $570 \mathrm{~nm}$ using a microplate absorbance reader (EXL 8008, Germany).

Determination of nitrite production. Inducible NO synthase (iNOS)-derived NO release is one of the major contributing factors during the inflammatory process in cerebral ischemic injury $(14,15)$. Nitrite production was assessed by the Griess reaction (16). In brief, each $50 \mu 1$ aliquot of the above-mentioned culture supernatants was collected and mixed with an equal volume of Griess reagent $[0.1 \%$ $\mathrm{N}$-(1-naphthyl)-ethylenediamine, $1 \%$ sulfanilamide in $5 \%$ phosphoric acid] and incubated at room temperature (RT) for $10 \mathrm{~min}$. Absorbance at $540 \mathrm{~nm}$ was measured in a microplate absorbance reader. Nitrite concentration was determined from a sodium nitrite standard curve.

Measurement of inflammatory cytokines. BV-2 cells were plated in a 24-well cell culture plate and incubated with GLGZD water extract $(50,100$ and $200 \mu \mathrm{g} / \mathrm{ml})$ in the presence or absence of LPS $(1 \mu \mathrm{g} / \mathrm{ml})$ for $24 \mathrm{~h}$. Following the manufacturer's instructions, a volume of $1 \mathrm{ml}$ of culture-medium supernatant was collected for measurement of the concentration levels of IL- 6 , TNF- $\alpha$ and IL- $1 \beta$ by the relevant ELISA kit.

Reverse transcription-polymerase chain reaction (RT-PCR). Total RNA was extracted from cultured microglial cells by using TRIzol reagent (Invitrogen, Carlsbad, CA, USA) and following the standard protocol. Purity and integrity of the RNA were assessed using a NanoDrop reader. Subsequently, first-strand cDNA synthesis was performed with $2 \mu \mathrm{g}$ total RNA using RevertAid ${ }^{\mathrm{TM}}$ H Minus First Strand cDNA Synthesis Kit (Fermentas, St. Leon-Rot, Germany) according to the manufacturer's instructions. The obtained cDNA was used to determine the mRNA levels of TNF- $\alpha$, IL-6, IL-1 $\beta$ and iNOS by a PCR kit (Fermentas). $\beta$-actin was used as an internal control.

Western blot analysis. BV-2 cells were plated overnight in 6 culture flasks and were then further incubated in serum-free medium for at least $4 \mathrm{~h}$ before treatments. After washing with cold PBS for three times, cells were lysed in RIPA buffer containing protease inhibitor PMSF (Roche Diagnostics, 

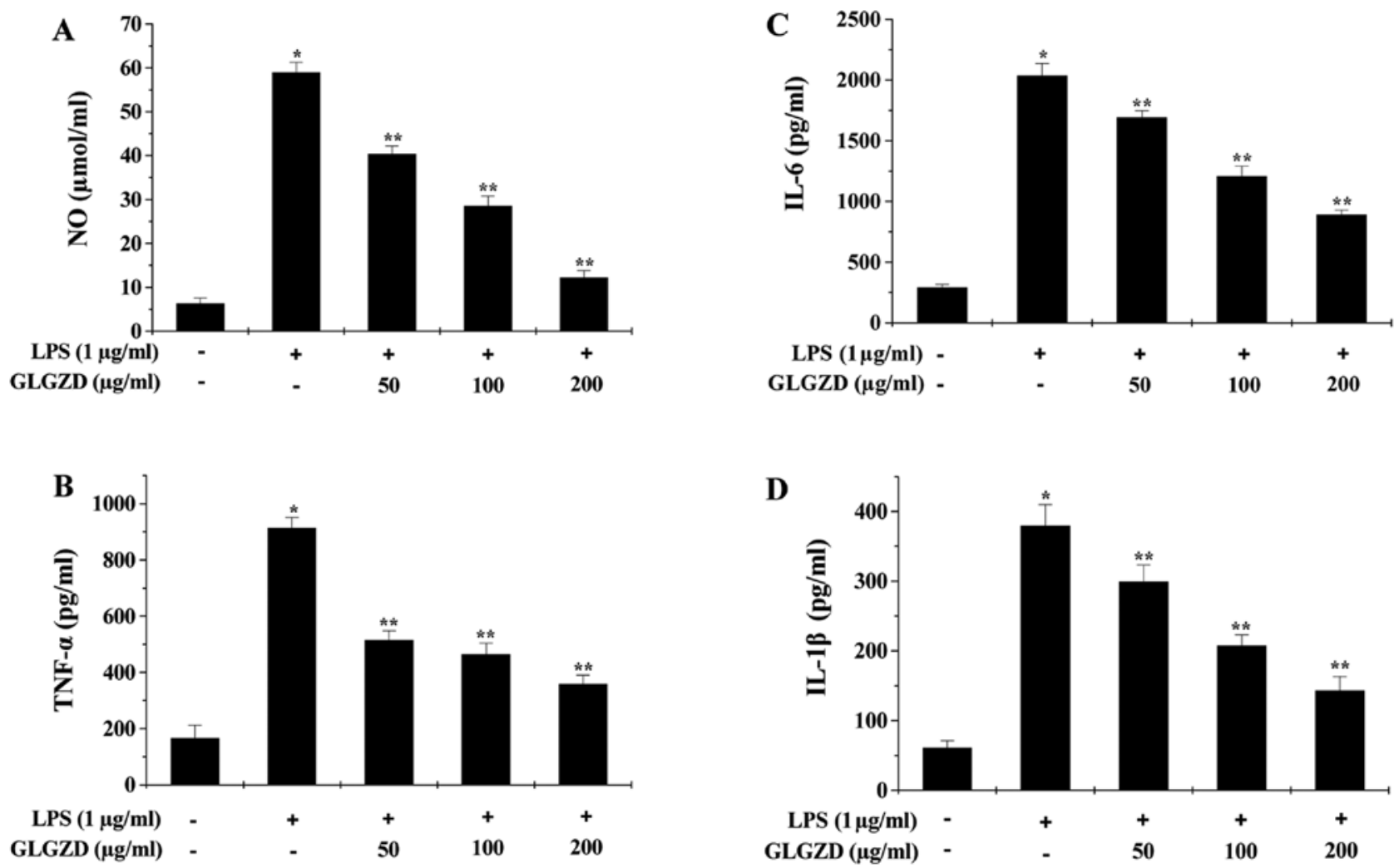

Figure 1. Effect of GLGZD treatment on the release of pro-inflammatory factors in LPS-stimulated microglial BV-2 cells. Cells were treated with LPS and/or the indicated concentrations of GLGZD. (A) Content of NO was measured using the Griess reaction. The secretion levels of (B) TNF- $\alpha$, (C) IL-6 and (D) IL-1 $\beta$ were measured using respective ELISA kits. Each value indicates the mean \pm SD from three independent experiments. "P<0.05 vs. untreated control cells; ${ }^{* *} \mathrm{P}<0.05$ vs. cells treated with LPS alone.

Mannheim, Germany). The content of the cells was pooled and centrifuged for $10 \mathrm{~min}$ at $12,000 \mathrm{x}$ g and stored at $-80^{\circ} \mathrm{C}$. The protein concentration of the lysates was measured using BCA quantification assay (Pierce, Rockford, IL, USA). Proteins $(50 \mu \mathrm{g})$ were separated using $12 \%$ (10\% for TLR4 analysis) SDS-PAGE and transferred to PVDF membranes with a $0.45-\mu \mathrm{m}$ pore size (IPVH00010; Millipore, Billerica, MA, USA). The membranes were incubated with primary antibodies overnight at $4^{\circ} \mathrm{C}$ : rabbit TLR4 monoclonal $\mathrm{Ab}$, rabbit

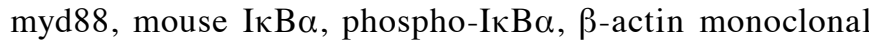
$\mathrm{Ab}(1: 1,000)$ diluted in immunoblot buffer (TBS containing $0.05 \%$ Tween-20 and 5\% non-fat dry milk). Following washing with TBST three times, membranes were incubated with the secondary antibody HRP-conjugated anti-mouse (or rabbit) $\operatorname{IgG}(1: 1,000)$ for $1 \mathrm{~h}$ at RT. After washing, the blots were detected with Chemiluminescence (ECL)-Plus (RPN2132; GE Healthcare Life Sciences, Pittsburgh, PA, USA) for $1 \mathrm{~min}$ using a camera along with the VersaDoc System (Bio-Rad, Hercules, CA, USA). The pixel intensities of the immunoreactive bands were quantified using the percentage adjusted volume feature of Quantity One 5.4.1 software (Bio-Rad). Data are expressed as a ratio of the intensity of the band of the protein of interest over the intensity of the band of the loading control protein ( $\beta$-actin).

Double-immunofluorescence labeling assay. For the detection of intracellular location of NF- $\mathrm{KB}$ p65, BV-2 cells were cultured on sterile glass coverslips and treated with GLGZD and LPS as described above. Following the various treatments, BV-2 cells were fixed with 4\% paraformaldehyde in PBS for $30 \mathrm{~min}$. After rinsing with PBS for three times, the cell membrane was permeabilized with $0.1 \%$ Triton $\mathrm{X}-100$ for $2 \mathrm{~min}$, and the coverslips with adherent cells were submitted to the doubleimmunofluorescence labeling assay. BV-2 cells were incubated with Hoechst (dilution 1:50,000; Sigma-Aldrich) plus NF-кB p65 (sc-8008, dilution 1:50; Santa Cruz Biotechnology, Inc.). The fluorescence signals were detected and analyzed using laser scan confocal microscopy (LSM 700; Carl Zeiss, Göttingen, Germany).

Statistical analysis. Data are expressed as means \pm SD. One-way ANOVA was used when comparing the data obtained from the different experimental conditions. In vitro experiments were conducted in triplicates; representative results are shown. A P-value of $<0.05$ was considered to indicate a statistically significant result.

\section{Results}

GLGZD inhibits the LPS-induced inflammatory response in microglial BV-2 cells. We first evaluated the effect of GLGZD on LPS-induced inflammation in BV-2 cells by measuring the production of $\mathrm{NO}$ and secretion levels of pro-inflammatory cytokines (TNF- $\alpha$, IL-6 and IL-1 $\beta$ ). As shown in Fig. 1, LPS stimulation for $24 \mathrm{~h}$ significantly induced the release of NO, TNF- $\alpha$, IL- 6 and IL- $1 \beta$, indicating an inflammatory response in BV-2 cells. However, the LPS-induced inflammation was significantly inhibited by GLGZD treatment in a 
A

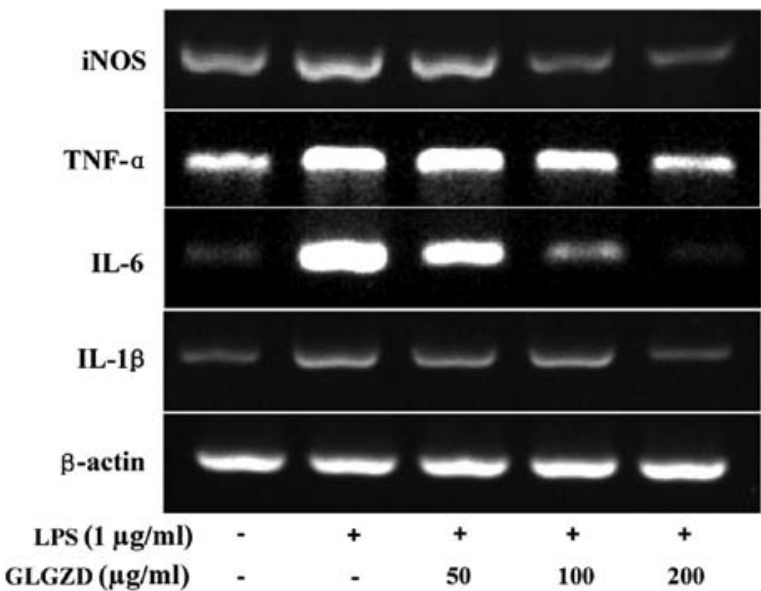

B

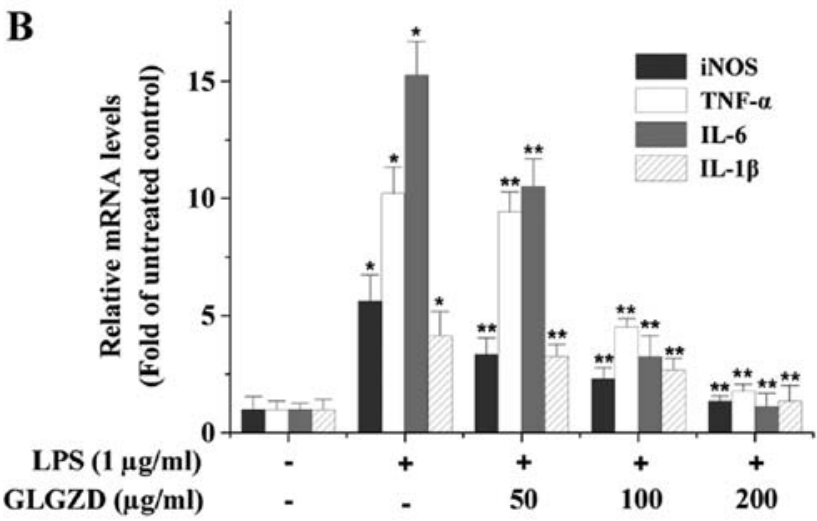

Figure 2. Effect of GLGZD on the transcription of pro-inflammatory factors in LPS-stimulated microglial BV-2 cells. (A) The mRNA expression of iNOS, TNF- $\alpha$, IL- 6 and IL- $1 \beta$ was determined by RT-PCR. $\beta$-actin was used as the internal control. Images are representatives of three independent experiments. (B) Densitometric analysis. The data were normalized to the mean mRNA expression of the untreated control. ${ }^{*} \mathrm{P}<0.05$ vs. untreated control cells; ${ }^{* *} \mathrm{P}<0.05$ vs. cells treated with LPS alone.

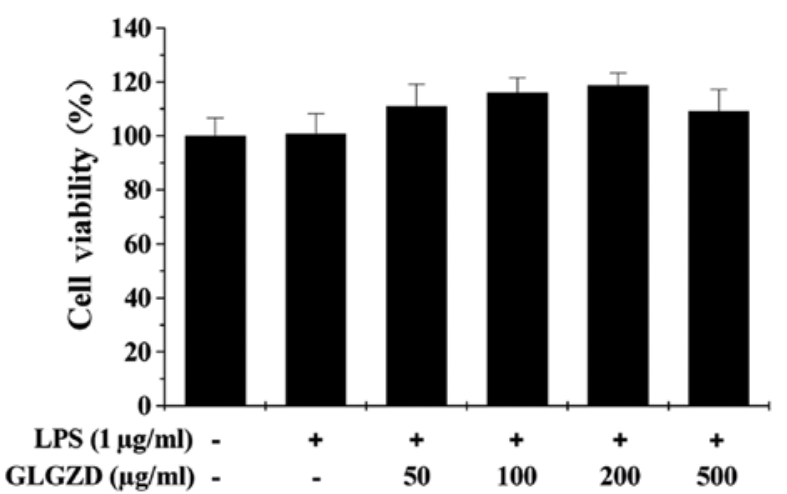

Figure 3. Effect of GLGZD and/or LPS on microglial BV-2 cell viability. Cells were treated with LPS and/or indicated concentrations of GLGZD for $24 \mathrm{~h}$. Cell viability was evaluated by MTT assay. The data were normalized to the viability of the untreated control cells. Each value indicates the mean \pm SD from at least three independent experiments.

dose-dependent manner. To further verify these observations, we determined the effect of GLGZD on the mRNA expression of the pro-inflammatory factors. As shown in Fig. 2, LPS stimulation profoundly increased the mRNA expression
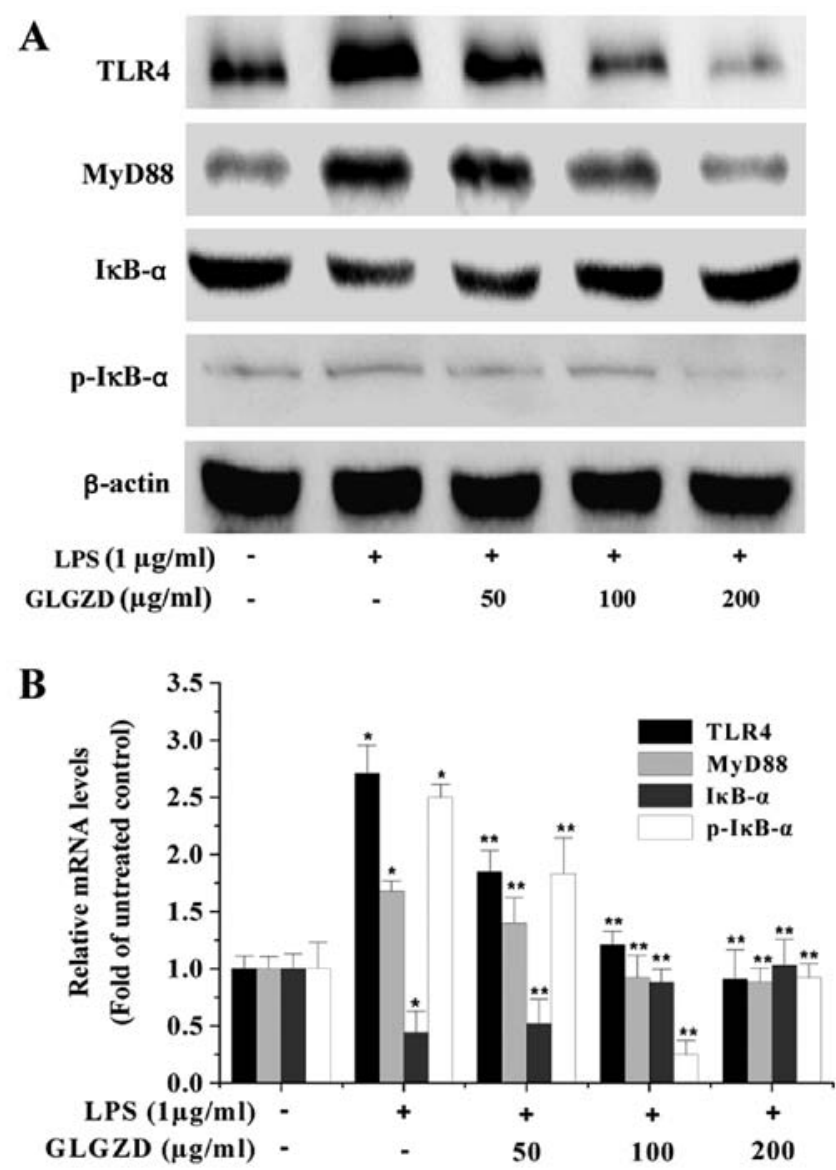

Figure 4. Effect of GLGZD on TLR4 and MyD88 protein expression and IкB phosphorylation in LPS-stimulated microglial BV-2 cells. (A) The protein expression levels of TLR4, MyD88 and the phosphorylation of I $\mathrm{B}$ were analyzed by western blotting. $\beta$-actin was used as the internal control. Images are representatives of three independent experiments. (B) Densitometric analysis. The data were normalized to the mean protein expression of the untreated control. ${ }^{*} \mathrm{P}<0.05$ vs. untreated control cells; ${ }^{* *} \mathrm{P}<0.05$ vs. cells treated with LPS alone.

of iNOS, TNF- $\alpha$, IL- 6 and IL-1 $\beta$ in BV-2 cells, which was dose-dependently and significantly suppressed by GLGZD treatment.

GLGZD does not display a cytotoxic effect in BV-2 cells. We next performed an MTT assay to evaluate the effect of GLGZD on cell viability. As shown in Fig. 3, treatment with various concentrations of GLGZD and/or LPS had no effect on the viability of the BV-2 cells, suggesting that inhibition of the LPS-induced inflammatory response in BV-2 cells did not result from the cytotoxic action of GLGZD.

GLGZD suppresses LPS-induced activation of the

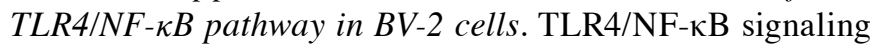
is one of the major pathways mediating immune and inflammation responses, which are involved in the expression of inflammatory mediators. The process of NF- $\mathrm{KB}$ activation includes several key links such as TLR4 activation, the presence of MyD88, phosphorylation/degradation of $\mathrm{I} \kappa \mathrm{B}$, and subsequent nuclear translocation of NF- $\mathrm{KB}$. To elucidate the mechanism of the anti-inflammatory activity of GLGZD, we examined its effect on the TLR4/NF-KB signaling pathway in 

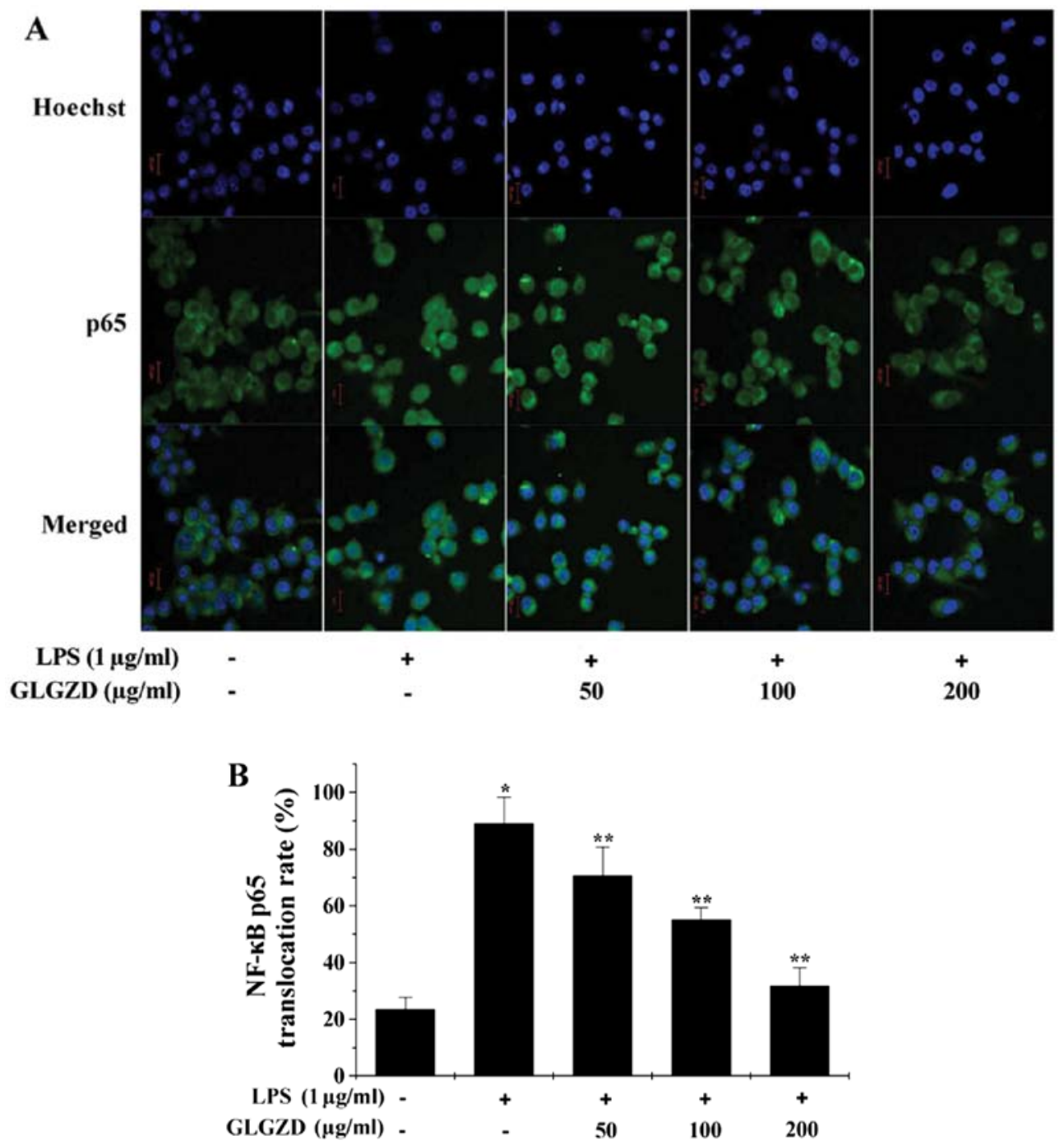

Figure 5. Effect of GLGZD on NF- $\kappa \mathrm{B}$ nuclear translocation in LPS-stimulated microglial BV-2 cells. (A) The NF- $\kappa \mathrm{B}$ p65 subunit was visualized by immunofluorescence staining (green), and the cells were counterstained with DAPI (blue). NF- $\kappa$ B nuclear translocation was represented by the co-localization of the p65 subunit with DAPI. Images were captured under a confocal fluorescence microscope with a magnification of $x 400$. Images are representative of three independent experiments. (B) The rate of NF- $\mathrm{B}$ p 65 nuclear translocation is expressed as the percentage of cells displaying co-localization of the p65 subunit with DAPI. Each value indicates the mean \pm SD from three independent experiments. ${ }^{*} \mathrm{P}<0.05$ vs. untreated control cells; ${ }^{* *} \mathrm{P}<0.05$ vs. cells treated with LPS alone.

microglial cells. As shown in Fig. 4, upon LPS stimulation, the expression of TLR4 and MyD88 as well as the phosphorylation level of I $\mathrm{B}$ were significantly increased. However, GLGZD significantly neutralized the effect of LPS stimulation, dosedependently reducing TLR4 and MyD88 protein expression and $\mathrm{I} \kappa \mathrm{B}$ phosphorylation levels in BV-2 cells. To further verify these results, we evaluated the effect of GLGZD on the nuclear translocation of NF- $\kappa \mathrm{B}$ which is a critical step for $\mathrm{NF}-\kappa \mathrm{B}$ activation. The NF- $\kappa \mathrm{B}$ p65 subunit was visualized by immunofluorescence staining. The cells were counterstained with DAPI, and NF- $\kappa \mathrm{B}$ nuclear translocation was recognized by the co-localization of the p65 subunit with DAPI. As shown in Fig. 5, LPS stimulation caused the nuclear translocation of the NF- $\kappa$ B p65 subunit, which was not observed in unstimulated cells. However, GLGZD treatment significantly blocked LPS-induced NF- $\kappa \mathrm{B}$ nuclear translocation in the BV-2 cells, in a dose-dependent manner. Collectively, these data suggest that the anti-inflammatory effect of GLGZD is mediated by suppression of TLR4/NF- $\mathrm{B}$ signaling in microglial BV-2 cells.

\section{Discussion}

Post-stroke neuroinflammation triggered by microglia cells is now believed to be a crucial mechanism leading to secondary injury of the CNS. Although an appropriate inflammatory response should be considered as a process to keep the CNS under dynamic surveillance, its injurious property has to be taken into account, including the release of pro-inflammatory mediators that are responsible for neurotoxic processes (17-19). Therefore, anti-inflammation therapy has been an attractive strategy to combat cerebral lesion. Gua Lou Gui Zhi decoction (GLGZD), a classical traditional Chinese medicine prescription, has been demonstrated to be effective for the clinic treatment of ischemic stroke. However, little is known about the mechanism of its neuroprotective action. 
Pro-inflammatory mediators produced in microglia, such as NO, TNF- $\alpha$, IL- 6 and IL- $1 \beta$, play an important role in neuro-inflammation; release of pro-inflammatory cytokines is therefore considered as an indicator for inflammatory response. Using LPS-stimulated microglial BV-2 cells as an in vitro inflammatory model of neural cells, in the present study we found that GLGZD significantly and dose-dependently reduced LPS-induced secretion of NO, TNF- $\alpha$, IL- 6 and IL- $1 \beta$, indicating that it inhibits the inflammatory reaction in neural cells. Neuro-inflammation is highly regulated by a family of pattern-recognition receptors, the Toll-like receptors (TLRs) (20-22). To date, over 13 members of the TLR family have been identified in mammals, of which TLR4 is the best studied (23-25). After activation via binding to specific ligands such as LPS, TLR4 mediates immune signals via recruitment of adaptor proteins (such as MyD88, Mal, TRIF and TRAM), resulting in the activation of transcription factors including $\mathrm{NF}-\kappa \mathrm{B}$. Under physiological conditions, NF- $\kappa \mathrm{B}$ is sequestered in the cytosol by $\mathrm{I} \kappa \mathrm{B}$ proteins via direct interaction. On the contrary, after cells receive pathological stimuli, IкB protein is phosphorylated, a process leading to its ubiquitination and degradation. Consequently, released NF- $\kappa \mathrm{B}$ translocates to the nucleus where it induces the expression of pro-inflammatory mediators (26). Using western blotting and immunofluorescence staining analyses, we found that GLGZD treatment significantly decreased the protein expression of TLR4 and MyD88, inhibited the phosphorylation of IkB and blocked the nuclear translocation of NF- $\mathrm{KB}$ in BV-2 cells, suggesting that GLGZD suppresses the activation of the TLR4/NF- $\kappa B$ signaling pathway.

In conclusion, we demonstrated for the first time that GLGZD exerts its anti-inflammatory actions via interference with TLR4/NF- $\mathrm{KB}$ signaling, which may be one of the mechanism whereby GLGZD ameliorates the damage in ischemic cerebral tissues.

\section{Acknowledgements}

This study was sponsored by the Guidance Project of the Fujian Provincial Department of Science and Technology (no. 2012D012), the Key Project of the Department of Health of Fujian Province (no. zlckf01), the Key Project of Fujian Provincial Department of Science and Technology (no. 2012Y0041) and the Project of Fujian Education Department (no. JA12176).

\section{References}

1. Donnan GA, Fisher M, Macleod M and Davis SM: Stroke. Lancet 371: 1612-1623, 2008.

2. Yenari MA, Kauppinen TM and Swanson RA: Microglial activation in stroke: therapeutic targets. Neurotherapeutics 7: 378-391, 2010.

3. Lehnardt S: Innate immunity and neuroinflammation in the CNS: the role of microglia in Toll-like receptor-mediated neuronal injury. Glia 58: 253-263, 2010.

4. Tambuyzer BR, Ponsaerts P and Nouwen EJ: Microglia: gatekeepers of central nervous system immunology. J Leukoc Biol 85: 352-370, 2009
5. Fan T, Jiang WL, Zhu J and Feng Zhang Y: Arctigenin protects focal cerebral ischemia-reperfusion rats through inhibiting neuroinflammation. Biol Pharm Bull 35: 2004-2009, 2012.

6. Park JS, Shin JA, Jung JS, et al: Anti-inflammatory mechanism of compound $\mathrm{K}$ in activated microglia and its neuroprotective effect on experimental stroke in mice. J Pharmacol Exp Ther 341: 59-67, 2012.

7. Park HY, Kim GY and Choi YH: Naringenin attenuates the release of pro-inflammatory mediators from lipopolysaccharidestimulated BV2 microglia by inactivating nuclear factor- $\kappa \mathrm{B}$ and inhibiting mitogen-activated protein kinases. Int J Mol Med 30: 204-210, 2012.

8. Mattson MP: NF- $\mathrm{KB}$ in the survival and plasticity of neurons. Neurochem Res 30: 883-893, 2005.

9. Wang J, Hou J, Zhang P, Li D, Zhang C and Liu J: Geniposide reduces inflammatory responses of oxygen-glucose deprived rat microglial cells via inhibition of the TLR4 signaling pathway. Neurochem Res 37: 2235-2248, 2012.

10. Dalloneau E, Pereira PL, Brault V, Nabel EG and Hérault Y: Prmt2 regulates the lipopolysaccharide- induced responses in lungs and macrophages. J Immunol 187: 4826-4834, 2011.

11. Sun X: Research on formula treating paralysis and spasticity From 'Treatise on febrile and miscellaneous diseases'. Zhongguo Zhong Yi Ji Chu Yi Xue Za Zhi 8: 644-645, 2010 (In Chinese).

12. Zhang L and Ai H: Effects of Gua Lou Gui Zhi Decoction on c-fos and c-jun on epileptic rats. Shi Yong Zhong Yi Yao Za Zhi 23: 21-22, 2005 (In Chinese).

13. Yang C, Chen L and Tao J: New usage of a classical formula - Gua Lou Gui Zhi Decoction. Liaoning J Tradit Chin Med 8: 166-167, 2012 (In Chinese).

14. Samdani AF, Dawson TM and Dawson VL: Nitric oxide synthase in models of focal ischemia. Stroke 28: 1283-1288, 1997.

15. Iadecola C: Bright and dark sides of nitric oxide in ischemic brain injury. Trends Neurosci 20: 132-139, 1997.

16. Kovac A, Erickson MA and Banks WA: Brain microvascular pericytes are immunoactive in culture: cytokine, chemokine, nitric oxide, and LRP-1 expression in response to lipopolysaccharide. J Neuroinflammation 8: 139, 2011.

17. McColl BW, Allan SM and Rothwell NJ: Systemic infection, inflammation and acute ischemic stroke. Neuroscience 158: 1049-1061, 2009.

18. McColl BW, Rothwell NJ and Allan SM: Systemic inflammatory stimulus potentiates the acute phase and CXC chemokine responses to experimental stroke and exacerbates brain damage via interleukin-1- and neutrophil-dependent mechanisms. J Neurosci 27: 4403-4412, 2007.

19. Jin R, Yang G and Li G: Inflammatory mechanisms in ischemic stroke: role of inflammatory cells. J Leukoc Biol 87: 779-789, 2010.

20. Beg AA: Endogenous ligands of Toll-like receptors: implications for regulating inflammatory and immune responses. Trends Immunol 23: 509-512, 2002.

21. Arroyo DS, Soria JA, Gaviglio EA, Rodriguez-Galan MC and Iribarren P: Toll-like receptors are key players in neurodegeneration. Int Immunopharmacol 11: 1415-1421, 2011.

22. Downes CE and Crack PJ: Neural injury following stroke: are Toll-like receptors the link between the immune system and the CNS? Br J Pharmacol 160: 1872-1888, 2010.

23. Lehnardt S, Lachance C, Patrizi S, et al: The Toll-like receptor TLR4 is necessary for lipopolysaccharide-induced oligodendrocyte injury in the CNS. J Neurosci 22: 2478-2486, 2002.

24. Lehnardt S, Massillon L, Follett P, et al: Activation of innate immunity in the CNS triggers neurodegeneration through a Toll-like receptor 4-dependent pathway. Proc Natl Acad Sci USA 100: 8514-8519, 2003.

25. Tanga FY, Nutile-McMenemy $\mathrm{N}$ and DeLeo JA: The CNS role of Toll-like receptor 4 in innate neuroimmunity and painful neuropathy. Proc Natl Acad Sci USA 102: 5856-5861, 2005.

26. Jung HW, Chung YS, Kim YS and Park YK: Celastrol inhibits production of nitric oxide and proinflammatory cytokines through MAPK signal transduction and NF- $\kappa \mathrm{B}$ in LPS-stimulated BV-2 microglial cells. Exp Mol Med 39: 715-721, 2007. 